\title{
ONTOLOGY-BASED QUESTION ANSWERING SYSTEM FOR AN ACADEMIC DOMAIN
}

\author{
García-Robledo Gabriela A, Reyes-Ortiz José A, \\ González-Beltrán Beatriz A and Bravo Maricela \\ Departamento de Sistemas, Universidad Autónoma Metropolitana, \\ Unidad Azcapotzalco, Mexico
}

\begin{abstract}
The development of question answering $(Q A)$ systems involves methods and techniques from the areas of Information Extraction (EI), Natural Language Processing (NLP), and sometimes speech recognition. A user interface that involves all these tasks requires deep development to improve the interaction between a user and a device. This paper describes a Spanish QA system for an academic domain through a multi-platform user interface. The system uses a voice query to be transformed into text. The semi-structured query is converted into SQWRL language to extract a system of ontologies from an academic domain using patterns. The answer of the ontologies is placed in templates classified according to the type of question. Finally, the answer is transformed into a voice. A method for experimentation is presented focusing on the questions asked in voice and their respective answers by experts from the academic domain in a set of 258 questions, obtaining a $92 \%$ accuracy.
\end{abstract}

\section{KEYWORDS}

Question answering systems, Speech recognition, Ontologies, SQWRL patterns, Information Extraction, Natural language processing.

\section{INTRODUCTION}

Teaching, research, and diffusion activities take place in an academic environment. Facilities are available, such as computer laboratories, libraries, classrooms, and auditoriums to realize these activities. In addition, actors participate in the activities to execute tasks. Administrative staff, professors, and students are some involved actors.

Ontologies allow to store and depict information about activities or events. Ontologies structure the information semantically to facilitate queries. However, the query about an event information and its related aspects (people, time, and physical spaces) involves a user request. Due to the large amount of information generated in the academic domain, this query process requires computational processing and a transformation into formal languages.

The answer extraction process from a voice query is a challenge today. The main objective of this paper is to extract an answer from an academic system of ontologies. For this reason, the whole process begins with a voice question from a user transformed into text and then represented in a semi-structured query using the procedure developed in [1]; the answer is placed in defined templates depending on the type of answer extracted; finally, the text with the final answer is converted from text to speech. This process involves a speech-to-text and text-to-speech translator, enriching a semi-structured query, and mapping entities to ontology elements. Also, David C. Wyld et al. (Eds): NLPTA, EDU, DSA, IoTE, VLSI, DPPR, ACITY, AIAA, CNDC - 2021 pp. 13-25, 2021. CS \& IT - CSCP 2021 
the identification of structural patterns (succession of structured elements frequently identified in different questions), the SQWRL execution query to get a specific response about events, physical spaces, and involved actors (people) that belong to the academic domain, and the generation of an answer understandable and friendly for the user is connected. The system of ontologies uses modules to consider people, time, and physical spaces; It is possible to answer questions such as who? where? and when? To make the query in voice format input and output, it uses Web Speech API in Java to run it in a multi-platform environment.

This paper aims to present a classification of templates based on different types of answers obtained from an information extraction method with an approach based on SQWRL patterns. As well, describe the development of a multiplatform user interface that has as input a voice query from a user from the academic domain, a transformation to an unstructured text, a natural language processing, extraction, and search of information in a system of ontologies, a representation of the answer in templates and voice output to the initial user.

The rest of the paper is organized as follows. Section 2 is focused on the most important and recent advances in the research areas of this paper. In Section 3, the proposed method for transforming the voice query into an SQWRL query is to extract information from the ontologies system and get an answer, which is returned to the user in the same voice format. The evaluation process is presented in Section 4, including experiments with 258 questions considering speechto-text and text-to-speech. Finally, Section 5 presents the conclusions and future work.

\section{RELATED WORK}

In this section, a review of the related work is discussed and presented.

A natural language interface is presented in [2] called FREYA that uses SPARQL searches on ontologies; it executes natural language request and generates an answer in the form of a graph, it offers the option of choosing between several options in case of ambiguity in the question to train the system. In [3], Ginseng uses static grammar rules that provide English structures and phrases. In [4] QuestIO System is developed, which is an interface without a predefined vocabulary. It uses a dictionary to identify classes, relations, instances, and property values based on ontology knowledge.

The system presented in [5] warns drivers about critical situations they may encounter on their journey; it is implemented with front and back cameras in their vehicle. The driver interacts with the system using only the voice. The driver observes a critical situation, activates the voice system, and describes the situation. Then, the system recognizes the voice of a driver, identifies the keywords, and transfers the information to a central system. The authors use ontologies that allow structuring semantic annotations by type of accident with the help of keywords and using indicators (green, yellow, and red) to know the priority with which it should be attended. In [6], a support system is developed for warehouse employees handling chemical materials using their hands and sight; it is implemented in devices so that with the help of voice recognition, they can record the tests without diverting attention from the sample. The voice records are made in an ontology, and the location is used to recognize what type of articles are used based on the knowledge of the ontologies.

A personal assistant is presented in [7] based on natural language processing, carried out through parse trees. The system receives a sentence from the user through voice; if it was understood satisfactorily, it notifies and then executes the query to the database, finally returning the answer the same way as the request. In [8], the system can simultaneously perform tasks to support a user by voice. Among the tasks it can handle are searching for a document or editing it, handling 
emails, and an activity schedule. The system is developed in multi-agent system architecture. In [9], a personal door assistant is implemented using voice; it allows a user to help manipulate the entrance to his office by acting as an intermediary between the visitor and the office owner. In [10], a portable personal assistant is patented to manipulate the contacts user, activities, and appointment scheduling using voice recognition. The system implements a voice user identifier to restrict access to personal data.

Furthermore, [11] developed a system to control several devices through applications within a house, known as Smart Home; the system uses voice to control devices and ontologies represent the information. The main aim is to execute specific tasks within the environment. The user requests and the dialogue system search the ontology to send the satisfactory operation to the Smart Home system. Later the extracted information is returned to the dialogue system, and it returns a report to the user as an answer. In [12], a system is presented that turns a space into an intelligent space for the service of the elderly to help them control electronic devices and different services using only the voice without the need for prior knowledge of the operation of the device.

There are works focused on people with limited vision, such as [13], whose search engine helps people with limited vision interact with a computer system in the same way as others, using only the voice. The visually impaired person communicates to the device with short sentences about what he/she requests on the internet, the system converts the voice sentences into text, and then it carries out the query to the ontologies that help to search the internet, the system displays the results of the URLs as the answer.

Finally, [14] also proposes a voice-manipulated search engine for blind people. This platform receives the voice with the user request, and it is converted into text. Then, the ontologies are consulted based on extracted keywords and thus support the search on the internet. The system returns the URLs that satisfy the requirement; they are converted to voice to answer the person who initiated the request. The authors comment that the platform helps in human-computer interaction with blind people. In [15], the QA system has input a query in natural language and an ontology, and as a response, it is extracted from the semantic markup of the compatible ontology. They propose a cascade architecture, where the query is translated into a set of triples compatible with the ontology. Furthermore, the natural language interface of [16] for the deduction of information stored in ontologies receives queries through voice in English. They developed a knowledge generator module (MGC) that answers user queries through the interface module (MI).

With the review of the work related to the disciplines involved in this paper, it is possible to observe the importance of carrying out an adequate transformation from a voice query into a structured representation and then using templates classification has been a transformation from text to speech.

\section{QA SYSTEM PROPOSED}

For the development of the QA System, a method was designed and implemented that transforms a query issued in natural language through a voice interface into a query in SWRL language; the answer is returned in the same input format. This process includes a voice input and output, a mapping of ontological entities, identifying structural patterns, executing an SQWRL query to obtain an answer from a system of ontologies, and classifying templates that allow better interaction between the user and the device, as in Figure 1 shows the architecture of the proposed Question-Answering System. 


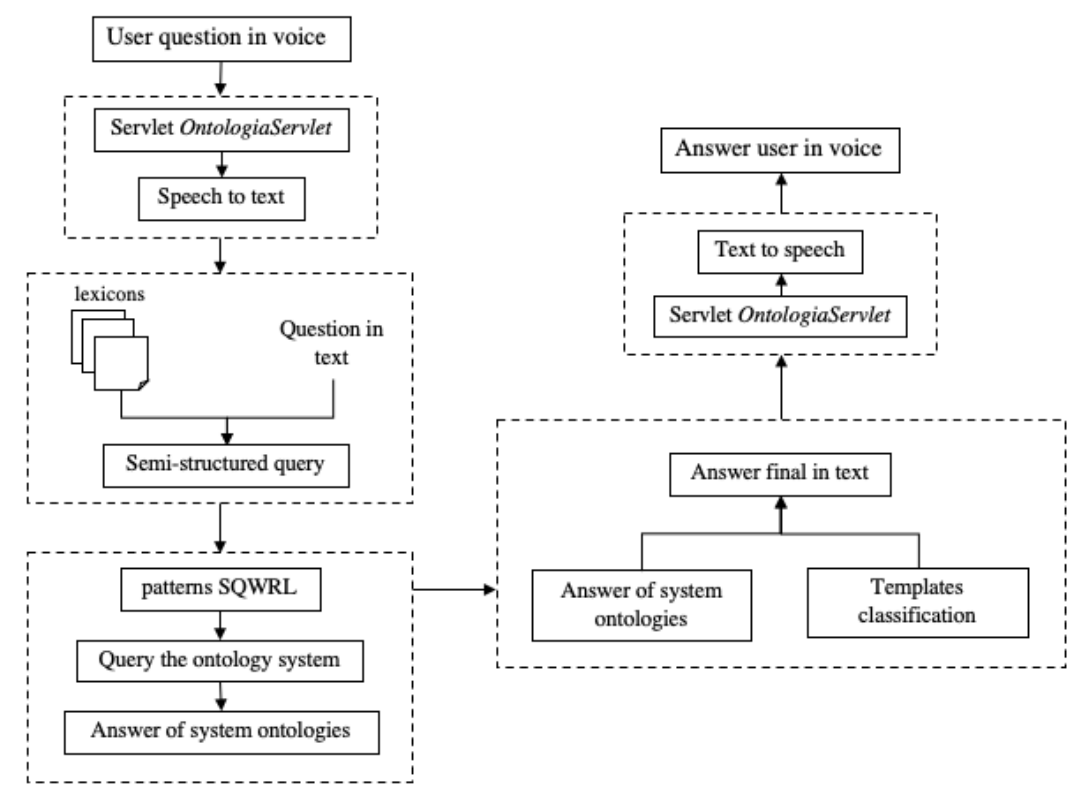

Figure 1. Proposed Question-Answering System Architecture

\subsection{Speech to text}

The user makes the question by voice. The Web Speech API in Java was used for speech recognition. JavaScript is used for the speech recognizer to transform the speech into text. When speech recognition is performed, it refers to a servlet named OntologiaServlet. The servlet names all the classes in charge of natural language processing and searching in the ontologies. The code is available at: https://github.com/UamAISII/AppVoz.git.

The answer generated in step 3 is used for the speech recognizer to transform the text to speech and is sent to the query interface.

\subsection{Query processing}

The query voice transformed into text is converted into a semi-structured query; this is created following the methodology reported in [1] by identifying entities and components of academic domain queries with a precision of $96 \%$. The method in [1] is made from lexicons of events, physical spaces, and people in an academic environment, available at: https://github.com/UamAISII/AppVoz.git. They analyzed a system of ontologies named Intelligent Environment and developed in [17], which contains Person, Event, Sensor Network, Physical Space, and Time. An information retrieval structure and an ontological tuple are created with a particular structure, preserving unstructured elements [1]. This semi-structured query is improved in [18] to consider elements necessary to answer the request.

The semi-structured query in [18] has various elements, such as the type of question and the set of relevant entities that the request contains. The relevant entities are those that contain the information necessary to respond to the query. This set of information is represented in a 5-tuple of elements. An answer from the system of ontologies is presented using enrichment to the structure of [1] and the identification of 3 structural patterns in SQWRL to query the ontology system. The method used in [18] has a precision of $96.8 \%$, and this is available at: https://github.com/UamAISII/AppVoz.git. 


\subsection{Answer generated}

The answer received from the SQWRL query is placed in templates with some elements of the ontological mapping for each type of question developed in [18], the representation shown in Equation 1.

$$
[O, S, P, T P, C, P 2, T P 2]
$$

Where:

- O is the ontology or ontologies to be consulted.

- $\mathrm{S}$ is the subject extracted from the question.

- $\mathrm{P}$ is the property of the subject, for example, if it's the name of a person, hasName is placed or if it's the name of an auditorium, hasNamePhysicalSpace is placed. These properties correspond to the model of the ontology system.

- TP is the DataProperty type or ObjectProperty type.

- $\mathrm{C}$ is the class of the subject contained in the queried ontology and contains the information to answer, for example, Professor, Building.

- $\quad \mathrm{P} 2$ is the property that is asked, for example, if we want to know the student ID, this data will contain hasStudentID.

- $\quad$ TP2 is the type of P2 and can be DataProperty or ObjectProperty.

In a Person question, the question is asked by name, employer ID (economic number), student ID, email, or category. If the question is for the category of a professor or student ID, the template is formed as follow:

$$
\begin{gathered}
\mathrm{La}+\mathrm{P} 2+d e+\mathrm{S}+e s+\text { ?res } \\
(\mathrm{The}+\mathrm{P} 2+\text { of }+\mathrm{S}+\text { is }+ \text { ?res })
\end{gathered}
$$

For example, the question is: ¿Cuál es la categoría de Pérez Pedro? (What is the category of Pérez Pedro?), the answer generated is: La categoría de Pérez Pedro es profesor titular $B$ (The category of Pérez Pedro is full professor B).

In the case of asking for the name, economic number, or email of a person, the answer is represented:

$$
\begin{gathered}
E l+\mathrm{P} 2 d e+\mathrm{S}+e s+\text { ?res } \\
(\mathrm{The}+\mathrm{S}+\mathrm{P} 2 \text { is }+ \text { ?res })
\end{gathered}
$$

For example, the question is: ¿Cuál es el correo de Pérez Pedro? (What is the e-mail of Pérez Pedro?). The answer generated is: El correo de Pérez Pedro es pperez@azc.uam.mx (The e-mail of Pérez Pedro is pperez@azc.uam.mx).

For questions of the physical space type, it must be analysed if Equation 1 contains the ObjectProperty constrainsPhysicalSpace in the position of TP2; if so, the answer is generated as follows:

$$
\mathrm{S}+\mathrm{P} 2+\text { ?res }
$$


For example, the question is: ¿Qué oficinas contiene el edificio C? (What offices does build C contain?). The answer generated is: Edificio $C$ contiene librería, auditorio Incalli Ixcahuicopa, recursos humanos, lenguas extranjeras, covi, cajas (Building $\mathrm{C}$ contains a library, Incalli Ixcahuicopa auditorium, human resources, languages, covi, cashier).

In case of not contain the ObjectProperty containsPhysicalSpace, the answer is:

$$
\begin{gathered}
\mathrm{S}+\text { está }+\mathrm{P} 2+\text { ?res } \\
(\mathrm{S}+\text { is }+\mathrm{P} 2+\text { ?res })
\end{gathered}
$$

For example, the question is: ¿Dónde está el auditorio F001? (Where is auditorium F001?), the answer generated is: Auditorio F001 está ubicado en edificio F planta baja (Auditorium F001 is in building $\mathrm{F}$, ground floor).

The event type question type has three different templates; these depend on the answers received in the SQWRL queries. If the question is for the description of an event, the answer is:

$$
\begin{aligned}
& \mathrm{S}+\text { trata de + ?res } \\
& (\mathrm{S}+\text { tries to + ?res })
\end{aligned}
$$

For example, the question is: De qué trata el taller paisajismo 101 (What is landscaping workshop 101 about?) the answer generated is: Taller paisajismo 101 trata de reflexionar sobre la importancia de la construcción y diseño; para ello es importante conocer los recursos lineamientos y criterios básicos que se manejan en espacios exteriores (Landscaping workshop 101 tries to reflect on the importance of construction and design; for this it is important to know the resources, guidelines and basic criteria that are handed in outdoor spaces).

If the question is to obtain the event date from its name, the answer is formed with the variables ?res and ?res2. The answer is:

$$
\begin{aligned}
& \mathrm{S}+\text { se realizará el }+ \text { ?res } 1+\text { de ?res } 2 \\
& (\mathrm{~S}+\text { will be held on }+ \text { ?res } 1+\text { ?res } 2)
\end{aligned}
$$

For example, the question is: ¿Cuándo es el taller de prevención de violencia contra las mujeres? (When is the workshop for the prevention of violence against women?), the answer generated is: Taller de prevención de violencia contra las mujeres se realizará el 17 de Agosto (Workshop for the prevention of violence against women will be held on August 17).

Also, it asks for the name of the event from a date, the answer is:

$$
\text { El nombre del evento es }+ \text { ?res }
$$

(The name of the event is + ?res)

For example, the question is: ¿Cuál es el nombre del congreso del 18 de junio? (What is the name of the congress on June 18?). The answer generated is: El nombre del evento es congreso de cohetes hidropropulsados (The name of the event is the hydro-powered rocket congress).

The questions of the event-person are the participants of an event from the date; in this case the answer is:

En el evento del $+\mathrm{S}+$ participa + ?res

(In the event on $+\mathrm{S}+$ participate + ?res) 
For example, the question is: ¿Quién participa en el congreso del 15 de enero? (Who participates in the congress on January 25th?). The answer is: En el evento del 15 de enero participa Pérez Pedro, Gómez María (In the event on January 15th participate Perez Pedro, Gómez María).

In the case of asking for the participants of an event with the name:

$$
\begin{gathered}
E n+\mathrm{S}+\text { participa }+ \text { ?res } \\
(\text { In the }+\mathrm{S}+\text { participate }+ \text { ?res })
\end{gathered}
$$

For example, the question is: ¿Quién participa en el seminario de fisica? (Who participates in the physics seminar?). The answer is: En seminario de física participa Pérez Pedro, Gómez María. (In the physics seminar participate Pérez Pedro, Gómez María).

The questions of the physical space-event type are classified in two. If the question is about the place where an event takes from the date, the answer is:

$$
\begin{aligned}
& \text { El evento del }+\mathrm{S}+\text { se realizará en }+ \text { ?res } \\
& (\text { The }+\mathrm{S}+\text { will be held in }+ \text { ?res })
\end{aligned}
$$

For example, the question is: ¿Dónde es el taller del 18 abril? (Where is the workshop April 18?). The answer is: El evento del 18 de abril se realizará en laboratorio E306 (The April 18 event will be held in laboratory E306).

Also, it asks for the name of the event; in this case the answer is represented:

$$
\begin{gathered}
\mathrm{S}+\text { se realizará en }+ \text { ?res } \\
(\mathrm{S}+\text { will be held in }+ \text { ?res })
\end{gathered}
$$

For example, the question is: Dime dónde es el taller de prevención de violencia contra las mujeres (Tell me where is the workshop for the prevention of violence against women). The answer is: Taller de prevención de violencia contra las mujeres se realizará en laboratorio Steve Jobs (Workshop for the prevention of violence against women will be held in the Steve Jobs laboratory).

The questions of the physical space - person type, can be by the person who is assigned to a workplace, the answer is:

$$
\begin{gathered}
E l+\mathrm{S}+\text { está asignado a }+ \text { ?res } \\
(\mathrm{S}+\text { is assigned to }+ \text { ?res })
\end{gathered}
$$

For example, the question is: ¿A quién le pertenece el cubículo H261? (Who owns cubicle H261?), the answer is: El cubículo H261 está asignado a Pérez Pedro (Cubicle H261 is assigned to Pérez Pedro).

Finally, when the question is about a person workplace based on their name or economic number, the answer is:

$$
\begin{gathered}
\mathrm{S}+\text { se encuentra en }+ \text { ?res } \\
(\mathrm{S}+\text { is in }+ \text { ?res })
\end{gathered}
$$


For example, the question is: ¿Cuál es el cubículo del profesor Pérez Pedro? (What is the cubicle of Professor Perez Pedro?), the answer is: Pérez Pedro se encuentra en cubículo H286 (Pérez Pedro is in cubicle H286).

\subsection{Query interface}

JSP (JavaServer Pages) technology connects the graphical query interface and the developed natural language processing.

The query interface is designed to help the user; when the query interface is opened, the user observes a window with a green button, as in Figure 2.

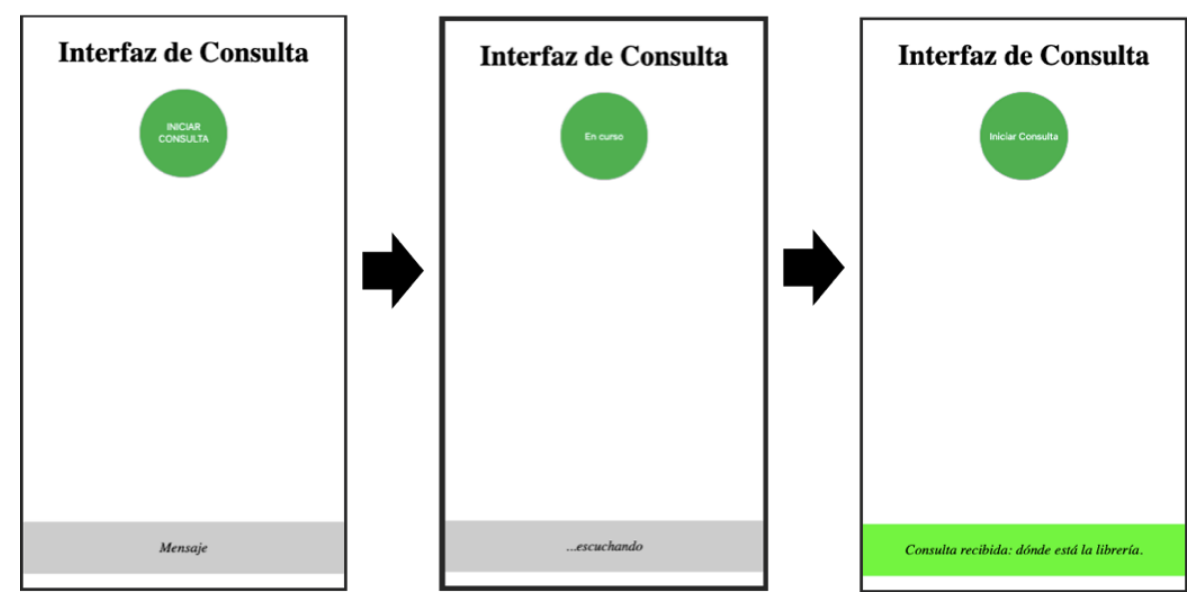

Figure 2. Query interface design

The user presses the Iniciar consulta (Start query) button and can begin to ask by voice; on the interface see a legend ...Escuchando (... listening) is shown to indicate that you can start talking and the button changes to En curso (In progress). As a final part, the friendly voice of the system mentions the answer in Spanish that it obtains from the ontology system and shows the query at the bottom, so the user can know it was recognized in the query interface. A use case is a bellow:

Use case "Juan wants to know where the library is":

When Juan presses the Iniciar consulta (start query) button, the system goes to the listening state and waits for the query. Juan asks by voice the question ¿Dónde está la librería? (Where is the library?). When the system listens for a long pause, it terminates the listening state and goes into an in-progress state. During this period, the system performs query processing and the answer generated.

If the system recognizes the query, it is displayed at the bottom as consulta recibida: dónde esta la librería (Query received: Where is the library). Moreover, the interface terminates the progress state and into a speaking state where the answer is heard through voice: La librería está en el edificio C planta baja (The library is in building C, ground floor). If it is not recognized, the answer is: Por el momento, no puedo responder (At the moment, I cannot answer). 


\section{EXPERIMENTATION AND RESULTS}

For evaluation purposes, a method for experimentation is assessed and executed. This method utilizes data that is extracted from an academic domain ontology [16]. This ontology contains 855 individuals, 91 classes, 10736 axioms, 36 object relations, and 51 properties or datatype relations. A total of 258 questions were defined by experts (research faculty members, students, and assistants), the language of the questions generated in Spanish. Seventy experts participated in defining the questions, specifically professors, assistants, and students. From these questions, a corpus was integrated. Experimentation method and corpus are available at: https://github.com/UamAISII/AppVoz.git.

The QA system developed can be used in mobile devices with two operating systems, such as Android or iOS. The precision of each question was evaluated manually by experts.

The speech recognizer has a margin of error due to different situations, such as ambient noise, user diction, or unknown words. It was considered part of the evaluation of whether the voice recognizer identified the complete question or if an absence of any word influences the system to answer correctly (partially identified question). For example, if the user asks ¿Dónde se encuentra la oficina del profesor Pedro Pérez? (Where is the office of Professor Pedro Pérez?), and the recognizer only identifies se encuentra oficina del profesor Pedro Pérez (is the office of Professor Pedro Pérez).

Table 1 shows speech recognition evaluation for the Person question-type. For this issue, 11 questions were asked: 79 questions were fully recognized, and 32 questions were wrong. In addition, out of 32 questions that were partially recognized, 27 correct responses were obtained, and of the 79 fully recognized questions, 74 successful responses were obtained.

Table 1. Person question-type speech recognition performance evaluation.

\begin{tabular}{|l|l|l|l|}
\hline Question voice recognition & Recognized questions & Correct answer & Wrong answer \\
\hline Fully identified & 79 & 74 & 5 \\
\hline Partially identified & 32 & 27 & 5 \\
\hline TOTAL & $\mathbf{1 1 1}$ & $\mathbf{1 0 1}$ & $\mathbf{1 0}$ \\
\hline
\end{tabular}

Nineteen questions of the PhysicalSpace question type were applied. In Table 2, it is observed that 14 questions were fully recognized, and five questions were not. Of the 14 fully recognized questions, $92.86 \%$ correct answers were obtained, and of the five partially recognized questions, all questions were answered successfully.

Table 2. PhysicalSpace question-type speech recognition performance evaluation.

\begin{tabular}{|l|l|l|l|}
\hline Question voice recognition & Recognized questions & Correct answer & Wrong answer \\
\hline Fully identified & 14 & 13 & 1 \\
\hline Partially identified & 5 & 5 & 0 \\
\hline TOTAL & $\mathbf{1 9}$ & $\mathbf{1 8}$ & $\mathbf{1}$ \\
\hline
\end{tabular}

Table 3 shows the speech recognition evaluation of the Event question type. For this type, 35 questions were completely identified, and five questions were not, of the 40 questions that were asked for this type. Of the five partially recognized questions, all were effectively obtained; and of the 35 fully identified questions, $88.57 \%$ of successful responses were obtained. 
Table 3. Event question-type speech recognition performance evaluation.

\begin{tabular}{|l|l|l|l|}
\hline Question voice recognition & Recognized questions & Correct answer & Wrong answer \\
\hline Fully identified & 35 & 31 & 4 \\
\hline Partially identified & 5 & 5 & 0 \\
\hline TOTAL & $\mathbf{4 0}$ & $\mathbf{3 6}$ & $\mathbf{4}$ \\
\hline
\end{tabular}

For the Person-PhysicalSpace composite question-type, eight questions were asked, five were fully recognized, and three were partially recognized. All questions were answered successfully. Table 4 shows the evaluation information for this type of question.

Table 4. Person-PhysicalSpace question-type speech recognition performance evaluation.

\begin{tabular}{|l|l|l|l|}
\hline Question voice recognition & Recognized questions & Correct answer & Wrong answer \\
\hline Fully identified & 5 & 5 & 0 \\
\hline Partially identified & 3 & 3 & 0 \\
\hline TOTAL & $\mathbf{8}$ & $\mathbf{8}$ & $\mathbf{0}$ \\
\hline
\end{tabular}

In the Person-Event composite question-type, 40 questions were asked, 34 were fully identified, and six partially. All fully identified questions were successfully answered, and partially identified questions obtained the $66.66 \%$ of correct answers. The results for this type of question are detailed in Table 5.

Table 5. Person-Event question-type speech recognition performance evaluation.

\begin{tabular}{|l|l|l|l|}
\hline Question voice recognition & Recognized questions & Correct answer & Wrong answer \\
\hline Fully identified & 34 & 34 & 0 \\
\hline Partially identified & 6 & 4 & 2 \\
\hline TOTAL & $\mathbf{4 0}$ & $\mathbf{3 8}$ & $\mathbf{2}$ \\
\hline
\end{tabular}

Table 6 shows the speech recognition evaluation for the Event-PhysicalSpace question-type. Thirty-six questions were fully recognized for this type, and four parts of the 40 questions were asked. Of the four identified questions, three were successfully obtained, and $94.44 \%$ of fully recognized questions obtained successful answers.

Table 6. Person-Physical space question-type speech recognition performance evaluation.

\begin{tabular}{|l|l|l|l|}
\hline Question voice recognition & Recognized questions & Correct answer & Wrong answer \\
\hline Fully identified & 36 & 34 & 2 \\
\hline Partially identified & 4 & 3 & 1 \\
\hline TOTAL & $\mathbf{4 0}$ & $\mathbf{3 7}$ & $\mathbf{3}$ \\
\hline
\end{tabular}

The graphic in Figure 3 shows the results of the evaluation. One hundred ninety-one questions were fully identified, and 47 questions were partially identified, both with a correct answer. For questions with an incorrect answer, 12 were fully recognized questions, and eight were partially identified. 


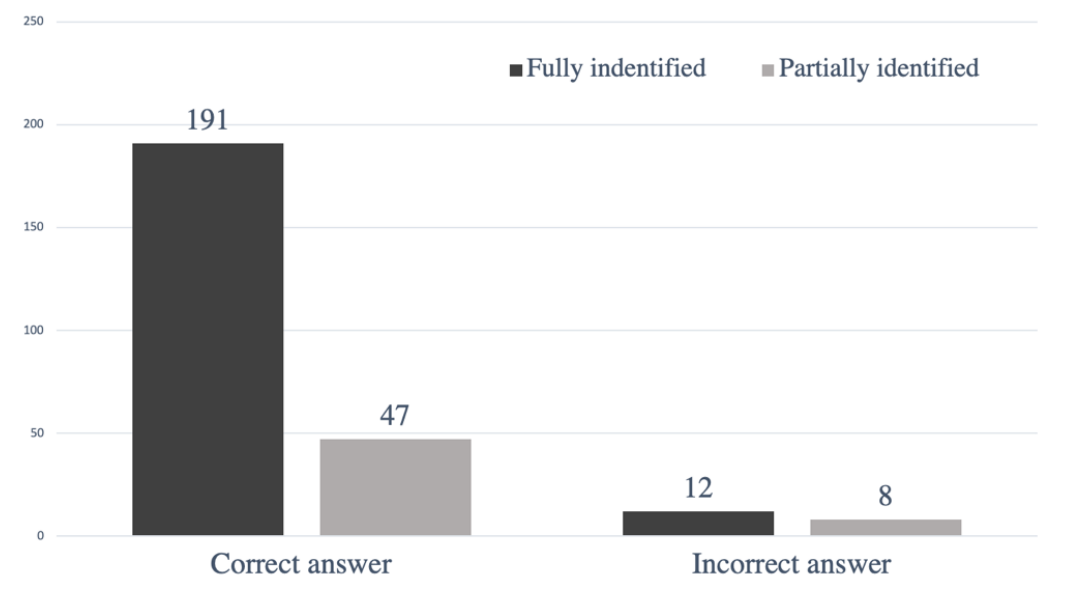

Figure 3. Global question-answer performance evaluation

The results of the global performance evaluation were obtained with the well-known metric in the Information Extraction area accuracy, shown in equation 2.

$$
P=(\text { Relevant Results }) /(\text { total questions })
$$

Where:

- Relevant results. Correct answers are fully identified, and correct answers are partially identified by the speech recognizer.

- Total questions. Total questions asked by experts (research faculty members, students, and assistants).

The results obtained were encouraging, achieving $92 \%$ of accuracy.

\section{Conclusions}

This paper has presented a QA system in the Mexican Spanish language within an academic environment. In addition, an ontology-based approach is described as an information representation model to perform queries in SQWRL from a voice query using structural patterns.

In the tests, promising results were obtained. The architecture of the QA system involves the following contributions: (a) developing a multi-platform voice query interface made with Web Speech API. (b) the construction of an ontological 5-tuple from natural language processing techniques in Spanish; (c) the identification and implementation of three structural patterns with syntax in the formal SQWRL language for the query in a system of ontologies of the academic domain; (d) the adaptation of an information extraction method based on lexicons in Spanish to a query architecture; (e) generating answer friendly to the user with templates selected according to the question type.

The results obtained in section 4 show that the QA system for voice is promising, having a more significant number of correct answers fully and partially identified. These results suggest an advance considering that they were carried out with the noise of an academic environment and the diction of different people who belong to the academic community. The question answering system showed that the number of correct answers is not affected by the partially recognized questions. 
As future work, (1) it is intended to check the functionality of the query interface in the Spanish language of Spain, to identify if the accent influences speech recognition; (2) enrich the semantic model in real-time; (3) adapt the query interface so that it can answer in real-time the location of the participants; (4) implementation of the QA system in another domain, for example, health;(5) Perform an approach of extraction and identification of answers to texts with deep learning algorithms and replace ontologies; (6) Carry out a measurement of the quality of the QA system with the mathematical model presented in [19] that predicts the degree of satisfaction of the interested parties $(\mathrm{Q})$ that constitute quality characteristics of the software.

\section{REFERENCES}

[1] García, G., Reyes, J., González \& B., Priego, (2019) “Extracción de información a partir de preguntas en español basada en lexicones", Academia Journals, Vol. 11, No. 8, pp1054-1059.

[2] Damljanovic, Danica, Agatonovic, Milan \& Cunningham, Hamish, (2010) "Natural Language Interfaces to Ontologies: Combining Syntactic Analysis and Ontology-based Lookup through the User Interaction". The Semantic Web: Research and Applications 7th Extended Semantic Web Conference, Vol. 6088, No. 1, pp106-120.

[3] Bernstein, Abraham, Kaufmann, Esther, Kaiser, Christian \& Kiefer, Christoph, (2006) "Ginseng: A guided input natural language search engine for querying ontologies", Jena User Conference.

[4] Tablan, Valentin, Damljanovic, Danica \& Bontcheva, Kalina, (2008) "A Natural Language Query Interface to Structured Information”, Proceedings of The Semantic Web: Research and Applications, Proceedings of the 5th European Semantic Web Conference, Vol. 5021, No. 1, pp361-375.

[5] Sosunova, Inna, Zaslavsky, Arkady, Theodoros, Anagnostopoulos, Alexey, Medvedev, Sergey, Khoruzhnikov \& Cladimir, Grudinin, (2015) "Ontology-based voice annotation of data streams in vehicles", Internet of Things, Smart Spaces, and Next Generation Networks and Systems, 15th International Conference, Vol. 9247, No. 1, pp152-162.

[6] Kopsa, Jirí, Míkovec, Zdenek \& Slavik, Pavel, (2005) “Ontology driven voice-based interaction in mobile environment". International Conference and Research Center for Computer Science.

[7] Rawassizadeh, Reza, Dobbins, Chelsea, Nourizadeh, Manouchehr, Ghamchili, Zahra \& Pazzani, Michael, (2017). "A natural language query interface for searching personal information on smartwatches". Pervasive Computing and Communications Workshops (PerCom Workshops), 2017 IEEE International Conference, pp679-684

[8] Paraiso, Emerson \& Barthès, Jean-Paul, (2006). "An intelligent speech interface for personal assistants in R\&D projects". Expert Systems with Applications, Vol. 31, No. 4, pp673-683.

[9] Yan, Hao \& Selker, Ted, (2000) "Context-aware office assistant". Proceedings of the 5th international conference on Intelligent user interfaces, pp276-279.

[10] Tsiao, J. C. S., Chao, D. Y. \& Tong, P. P., (2007) "Natural-Language Voice-Activated Personal Assistant" U.S. Patent No. 7,216,080. Washington, DC: U.S. Patent and Trademark Office.

[11] Huang, Cheng-Chi., Liu, Alan \& Zhou, Pei-Chuan, (2015) "Using Ontology Reasoning in Building a Simple and Effective Dialog System for a Smart Home System”, Systems, Man, and Cybernetics (SMC), 2015 IEEE International Conference, pp1508-1513.

[12] Portet, Francois, Vacher, Michel, Golanski, Caroline \& Meillon, Brigitte, (2012) "Design and evaluation of a smart home voice interface for the elderly: acceptability and objection aspects", Personal and Ubiquitous Computing, Vol. 17, No. 1, pp127-144.

[13] Bukhari, Ahmad \& Kim, Yong-Gi, (2012) "Ontology-assisted automatic precise information extractor for visually impaired inhabitants". Artificial Intelligence Review, Vol. 38, No. 1, pp9-24.

[14] Karthik, N., Ashwini, M. \& Anitha, K., (2014) "Voice Enabled Ontology Based Search Engine on Semantic Web For Blind", International Journal of Computer Science \& Engineering Technology, Vol. 5, No. 04, pp341-344.

[15] Lopez, Vanessa, Pasin, Michele \& Motta, Enrico, (2005) "Aqualog: An ontology-portable question answering system for the semantic web", European Semantic Web: Research and Applications Second European Semantic Web Conference, Vol. 3532, No. 1, pp546-562.

[16] Solís, Alejandro, Florencia, Rogelio, Acosta, Carlos \& López, Francisco, (2018) “Interfaz de lenguaje natural para deducción de información almacenada en ontologías”. Research in Computing Science, Vol. 147, No. 6. 
[17] Padilla, Josué, (2019) “Detección y representación de eventos en un ambiente académico inteligente", Master's Thesis, Universidad Autónoma Metropolitana, Unidad Azcapotzalco.

[18] Gabriela A. García-Robledo, Beatriz A. González-Beltrán, José A. Reyes-Ortiz \& Maricela Bravo, (2020) "Extracción de respuestas a partir de ontologías utilizando patrones estructurales en SQWRL", Research in Computing Science, Vol. 149, No. 8, pp571-585.

[19] Gheisari, M., Panwar, D., Tomar, P., Harsh, H., Zhang, X., Solanki, A., Nayyar, A. \& Alzubi, J., (2019). "An optimization model for software quality prediction with case study analysis using MATLAB”. IEEE Access, Vol. 7, pp 85124-85138.

\section{AUTHORS}

Gabriela A. García-Robledo is a partial-time researcher-professor at the Universidad Autónoma Metropolitana. She received a master's in computer science from Universidad Autónoma Metropolitana in 2020. Her current research interests include natural language processing, speech recognition and information extraction.

Jose A. Reyes-Ortiz received a Ph.D. in Computer Science from National Centre for Research and Technological Development, Cuernavaca, Mexico in 2013. Currently, he is a full-time researcher-professor at Universidad Autónoma Metropolitana. His current research interests include natural language processing, pattern recognition from text, and computational linguistics.

Beatriz A. González-Beltrán is a professor of Computer Science at the Universidad Autónoma Metropolitana. Her work focuses on artificial intelligence in human computer interaction. Beatriz completed her Ph.D. in the Institute of Engineering at the Univ. Grenoble Alpes, France.

Maricela Bravo obtained the degree of Doctor of Computer Science at the National Centre for Technological Research and Development (CENIDET) in 2006. She currently works as a full-time professor at the Autonomous Metropolitan University. Her research areas include knowledge representation, logical reasoning, distributed systems based on REST architectures, and intelligent systems for healthcare applications.
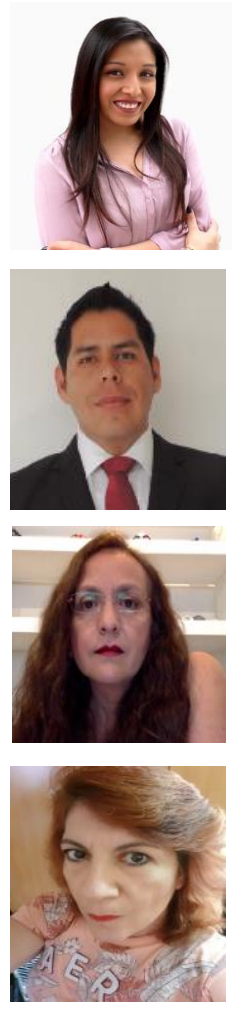

(C) 2021 By AIRCC Publishing Corporation. This article is published under the Creative Commons Attribution (CC BY) license. 